\title{
Selbstständige Ausübung von Heilkunde durch Pflegekräfte
}

Gertrud Ayerle, Gero Langer und Gabriele Meyer

\subsection{Hintergrund - 180}

14.1.1 Empfehlungen aus Gremien der Politikberatung - 180

14.1.2 Definition der Heilkunde - 181

14.1.3 Gesetzliche Grundlagen und Richtlinie - 181

14.1.4 Forschungsstand zur Substitution heilkundlicher Tätigkeiten - 182

14.2 Studiengang Evidenzbasierte Pflege - 184

14.2.1 Entwicklung und Umsetzung - 184

14.2.2 Qualitätssicherung - 185

14.3 Einmündung in die Gesundheitsversorgung - 185 Literatur - 187 


\section{- Zusammenfassung}

In Europa und international unterliegt die Aufgabenverteilung im Gesundheitswesen einschlägigen Transformationsprozessen. Die Kompetenzen Pflegender werden erweitert und Pflegende übernehmen definierte Tätigkeiten, die vormals Ärztinnen und Ärzten vorbehalten waren. Auch in Deutschland ist mit $\$ 63$ Abs. 3c SGB V seit zehn Jahren die Möglichkeit eröffnet, in Modellprojekten die selbstständige Ausübung von Heilkunde durch Berufsangehörige der Kranken- und Altenpflege zu erproben. Bislang gibt es jedoch erst an einem Standort in Deutschland ein Modellprojekt, das zwei Bereiche (Diabetes Typ 2 und chronische Wunden) aus den Richtlinien des Gemeinsamen Bundesausschusses auf Pflegende im Modellstudiengang „Evidenzbasierte Pflege" überträgt. Die Herausforderung besteht nun darin, die Absolventinnen und Absolventen mit entsprechenden beruflichen Rollen in die stationäre und ambulante Pflegepraxis zu integrieren und regulatorische Barrieren zu beseitigen.

In Europe and internationally, the allocation of tasks in the health care system is subject to transformation processes. Nurses' competencies are expanded and nurses substitute certain tasks that were previously carried out by physicians only. In Germany, a law was passed ten years ago that allows members of the nursing and elderly care profession to substitute physicians' tasks within the framework of pilot projects. Up to now, however, only one pilot project has been initiated. The $\mathrm{Ba}$ chelor degree programme „Evidence-based Nursing" prepares nurses to provide - apart from nursing care - comprehensive treatment for patients with diabetes type 2 and chronic wounds. The challenge now is to integrate graduates with appropriate professional roles into inpatient and outpatient nursing practice and to remove regulatory barriers.

\subsection{Hintergrund}

Die Rollen, die Pflegende einnehmen, sind soziokulturell, sozioökonomisch und durch die Arbeitsteilung im Gesundheitssystem bedingt.
Diese Arbeitsteilung sieht in anderen Ländern ganz anders aus: Beispielsweise praktizieren in den USA 40,5 Nurse Practitioners (NPs) pro 100.000 Einwohner, was ca. einem Fünftel der tätigen Ärztinnen und Ärzten entspricht. Auch in den Niederlanden, Kanada, Australien, Neuseeland und Irland praktizieren NPs (Maier et al. 2016); sie übernehmen dort breite Aufgaben im Gesundheitswesen und können die niedergelassenen Ärzte mit allen Kompetenzen ersetzen.

Auch in Deutschland ist mit $\$ 63$ Abs. 3c SGB V seit zehn Jahren die Möglichkeit eröffnet, in Modellprojekten die selbstständige Ausübung von Heilkunde durch Berufsangehörige der Kranken- und Altenpflege zu erproben. Bislang gibt es jedoch erst an einem Standort in Deutschland ein Modellprojekt, das zwei Bereiche aus den Richtlinien des Gemeinsamen Bundesausschusses (Bundesministerium für Gesundheit 2012) auf Pflegende im Modellstudiengang „Evidenzbasierte Pflege“ überträgt (Meyer 2018).

\subsubsection{Empfehlungen aus Gremien der Politikberatung}

Seit mehr als zehn Jahren fordert der Sachverständigenrat zur Begutachtung der Entwicklung im Gesundheitswesen die Entwicklung neuer Formen der Kooperation und Aufgabenteilung zwischen den Gesundheitsberufen (Sachverständigenrat (SVR) 2007, 2014). Angesichts der zunehmenden Bedarfe von Menschen ab 65 Jahren, die häufig an mehreren Erkrankungen und Einschränkungen leiden (Multimorbidität), ist die „adäquate Qualifizierung und Vorbereitung auf die veränderten Nutzerrealitäten eine der wesentlichen Anforderungen an alle Gesundheitsberufe“ (SVR 2007). Dabei ist die „Notwendigkeit, die Arbeitsverteilung zwischen den Berufen im Gesundheitswesen anzupassen“ (SVR 2007), eine der zentralen Anforderungen an das Case Management sowohl in den Bereichen der ambulanten, stationären und rehabilitati- 
ven Versorgung als auch transsektoral, deren Schnittstellen übergreifend.

Der Wissenschaftsrat schlug vor, Pflegende künftig auch an Universitäten bzw. deren Medizinischen Fakultäten auszubilden und ihnen sowohl die Berufszulassung als auch einen Bachelorabschluss zur unmittelbaren Tätigkeit an Patientinnen und Patienten zu ermöglichen (primärqualifizierender Studiengang). Vom Setting der Ausbildung abgesehen muss das maßgebliche Ziel angestrebt werden, die Auszubildenden mit erweiterten Fach-, Methoden-, Kommunikations-, Sozial-, Selbstund (Fall-)Management-Kompetenzen auszustatten, um sie als Reflective Practitioner zur Pflege von Patienten mit komplexem Krankheitsgeschehen zu befähigen (Wissenschaftsrat 2012).

Die Deutsche Hochschulmedizin e. V., die mehrere universitäre und universitär-klinische Verbände vertritt, forderte 2014 insbesondere akademisch qualifiziertes Pflegepersonal für die berufsgruppenübergreifenden Teams in den Universitätsklinika. Dabei verwies sie auf die dort vorhandene Expertise und die Möglichkeit, Synergien zwischen dem Medizinund dem Pflegestudiengang sowie weiteren Gesundheitsberufen zu nutzen. Eine gezielte und ausreichende finanzielle Förderung sei dafür jedoch unverzichtbar (Deutsche Hochschulmedizin e. V. 2014).

\subsubsection{Definition der Heilkunde}

Im einschlägigen $₫ 28$ SGB V findet der Begriff Heilkunde keine Verwendung; es wird die ärztliche Behandlung beschrieben als „die Tätigkeit des Arztes, die zur Verhütung, Früherkennung und Behandlung von Krankheiten nach den Regeln der ärztlichen Kunst ausreichend und zweckmäßig ist. Zur ärztlichen Behandlung gehört auch die Hilfeleistung anderer Personen, die von dem Arzt angeordnet und von ihm zu verantworten ist. “1

1 Die Ausübung der Heilkunde, worunter im Gesetz die „Feststellung, Heilung oder Linderung von
Wenn Pflegende heilkundliche Tätigkeiten ausführen, übernehmen sie im Sinne der Substitution definierte Verantwortungsbereiche sowie die fachliche, wirtschaftliche und rechtliche Verantwortung dafür (Igl 2010). In letzterem Punkt unterscheidet sich die Substitution vom Prinzip der Delegation, bei dem die Pflegenden im Auftrag einer Ärztin oder eines Arztes zwar heilkundliche Tätigkeiten übertragen bekommen, die Ärztin oder der Arzt jedoch in der ärztlichen und juristischen Verantwortung bleibt (Igl 2010).

Die Diagnostik, Differenzialdiagnostik und Therapie(-festlegung), die Kernbereiche des Arztberufes darstellen, sind von einer Übertragung auf nicht-ärztliches Personal ausgeschlossen. Wenn jedoch die ärztliche Diagnose und Indikation feststehen, die die zwingende Voraussetzung für die Substitution sind, übernimmt die bzw. der Pflegende - neben der Verantwortung für die Ausübung von Maßnahmen - auch die Entscheidungsbefugnis, ob und in welchem Umfang die selbstständige Ausübung der Heilkunde medizinisch geboten ist.

\subsubsection{Gesetzliche Grundlagen und Richtlinie}

Im Jahr 2008 wurde infolge des Pflegeweiterentwicklungsgesetzes (2008) sowohl eine Erweiterung des $₫ 63$ SGB V als auch des $\$ 4$ Abs. 7 KfPflG (2003) vorgenommen. Letztere ermöglicht seither Modellvorhaben nach $₫ 63$ Abs. 3c SGB V, die die Vermittlung von Kompetenzen zur Ausübung heilkundlicher Tätigkeiten zum Ziel haben. Damit können so qualifizierte Pflegende im Sinne der Substitution heilkundliche Tätigkeiten übernehmen. Neben dem Gebot einer entsprechenden Qualifizierung ist darin vorgesehen, dass der Gemeinsame Bundesausschuss in einer Richtlinie Vorgaben macht, in welchen fachlichen Bereichen eine Übertragung von heilkundlichen

Krankheiten, Leiden oder Körperschäden bei Menschen" verstanden wird, ist auch Heilpraktikerinnen und Heilpraktikern erlaubt (HeilprG. 1939). 
Tätigkeiten erfolgen kann. Die Richtlinie, die 2012 veröffentlicht wurde, nimmt eine doppelte Perspektive auf heilkundliche Tätigkeiten ein, und zwar „diagnosebezogen“ und "prozedurenbezogen“. Festgelegt wurden die Diagnosen Diabetes mellitus Typ 1, Diabetes mellitus Typ 2, Chronische Wunden (z. B. Ulcus cruris), (Verdacht auf) Demenz (nicht palliativ) sowie (Verdacht auf) Hypertonus (ohne Schwangerschaft). Bei den Prozeduren wurden übertragbare ärztliche Tätigkeiten wie Infusionstherapie und Injektionen, Stomatherapie, Tracheostoma-Management, Anlage und Versorgung einer Magensonde, Versorgung und Wechsel eines suprapubischen Blasenkatheters, Schmerztherapie und -management, Patienten-, Case-, Überleitungsmanagement und psychosoziale Versorgung definiert (Gemeinsamer Bundesauschuss (G-BA) 2012).

Bislang hat die Medizinische Fakultät der Martin-Luther-Universität Halle-Wittenberg (MLU) das Alleinstellungsmerkmal, ein Modellvorhaben nach $₫ 63$ Abs. 3c SGB V im Rahmen eines primärqualifizierenden Pflegestudiengangs umzusetzen. Gründe für die Zurückhaltung anderer Universitäten und Ausbildungszentren könnten in den Anforderungen des KrPflG (2003) liegen, erstens die Vereinbarkeit der Ausbildung mit der Richtlinie 2005/36/EG zu gewährleisten (entsprechend der KrPflAPrV 2003), zweitens das Curriculum vom Bundesministerium für Gesundheit im Einvernehmen mit dem Bundesministerium für Familie, Senioren, Frauen und Jugend genehmigen zu lassen, drittens ein auf die Vermittlung von heilkundlichen Tätigkeiten bezogenes Modellvorhaben nach $\$ 63$ Abs. 3c SGB V zu vereinbaren und viertens eine zusätzliche (staatliche) Prüfung der erweiterten Kompetenzen vorzusehen (KfPflG 2003).

\subsubsection{Forschungsstand zur Substitution heilkundlicher Tätigkeiten}

Die Übertragbarkeit von Modellen der Substitution heilkundlicher Tätigkeiten aus anderen Ländern ist schwierig, denn zu speziell sind oft die Ausbildungen, Kompetenzen, Versorgungsstrukturen, Vergütungsregelungen und gesetzlichen Vorgaben.

In einem kürzlich aktualisierten CochraneReview (Laurant et al. 2018) wurden die Auswirkungen der Substitution ärztlicher Tätigkeiten durch Pflegende untersucht. Von insgesamt 18 eingeschlossenen randomisierten kontrollierten Studien wurde eine Studie in einem Land mit mittlerem Einkommen durchgeführt, alle anderen Studien in Ländern mit hohem Einkommen. Die Aufgaben und Kompetenzen der Pflegenden waren häufig unklar oder variierten zwischen und teilweise sogar innerhalb der Einzelstudien. In den Studien wurden vor allem Pflegende untersucht, die an der Erstversorgung (einschließlich der Notfallversorgung), der laufenden Betreuung bei körperlichen Beschwerden und der Nachsorge von $\mathrm{Pa}$ tientinnen und Patienten mit besonderen chronischen Erkrankungen wie Diabetes mellitus beteiligt waren.

In vielen Studien konnten die Pflegenden bei Bedarf zusätzliche Unterstützung oder Beratung durch Ärztinnen oder Ärzte erhalten. Insgesamt ist die Substitution im Bereich der Prävention und der Gesundheitserziehung in der Grundversorgung weniger gut untersucht.

Die Studienergebnisse legen nahe, dass eine pflegerische Versorgung im Vergleich zur ärztlichen Versorgung bei zahlreichen Erkrankungen wahrscheinlich ähnliche oder bessere Ergebnisse erzielt (vgl. • Tab. 14.1).

Die Auswirkungen der pflegerischen Betreuung auf die Inanspruchnahme von Leistungen sind gemischt: Durchschnittlich dauerten Konsultationen in der von Pflegenden geleiteten Primärversorgung 39 \% länger als bei Ärztinnen oder Ärzten und die Zahl der erneuten Konsultationen war bei Pflegenden 1,2-mal 
- Tabelle 14.1 Studienlage zur Substitution ärztlicher Tätigkeiten durch Pflegende

\begin{tabular}{|c|c|c|c|c|}
\hline Quelle & $\begin{array}{l}\text { Studien und } \\
\text { Teilnehmer }\end{array}$ & Effekte & $\begin{array}{l}\text { Evidence-Level } \\
\text { (GRADE) }\end{array}$ & Interpretation \\
\hline \multirow[t]{6}{*}{$\begin{array}{l}\text { Laurant et al. } \\
\text { (2018) }\end{array}$} & $\begin{array}{l}8 \text { Studien } \\
36.529 \text { Teil- } \\
\text { nehmer }\end{array}$ & $\begin{array}{l}\text { Mortalität: Relatives Risiko } \\
0,77 \text { mit } \mathrm{Cl}_{95 \%} \text { von } 0,57 \text { bis } \\
1,03\end{array}$ & Niedrig & $\begin{array}{l}\text { Die pflegerische Grund- } \\
\text { versorgung bedingt bei } \\
\text { bestimmten Patienten- } \\
\text { gruppen im Vergleich zur } \\
\text { ärztlichen Versorgung } \\
\text { einen Trend zu weniger } \\
\text { Todesfällen. }\end{array}$ \\
\hline & \multirow[t]{3}{*}{$\begin{array}{l}8 \text { Studien } \\
36.529 \\
\text { Teilnehmer }\end{array}$} & $\begin{array}{l}\text { Blutdruck systolisch: Mittel- } \\
\text { wertdifferenz }-3,73 \mathrm{mmHg} \\
\text { mit } \mathrm{Cl}_{95 \%}-6,02 \text { bis }-1,44\end{array}$ & \multirow[t]{3}{*}{ Mittel } & \multirow{3}{*}{$\begin{array}{l}\text { Der Blutdruck war in der } \\
\text { von Pflegenden } \\
\text { geleiteten } \\
\text { Primärversorgung leicht } \\
\text { verbessert, ebenso wie } \\
\text { Schmerzen bei Patienten } \\
\text { mit Rheuma, } \\
\text { Körperfunktionen und } \\
\text { Cholesterol-Spiegel. }\end{array}$} \\
\hline & & $\begin{array}{l}\text { Blutdruck diastolisch: } \\
\text { Mittelwertdifferenz } \\
-2,54 \text { mmHg mit } \mathrm{Cl}_{95 \%} \\
-4,57 \text { bis }-0,52\end{array}$ & & \\
\hline & & $\begin{array}{l}\text { HbA1c-Spiegel: Mittelwert- } \\
\text { differenz 0,08 mit } \mathrm{Cl}_{95 \%} \\
-0,25 \text { bis } 0,41\end{array}$ & & \\
\hline & $\begin{array}{l}7 \text { Studien } \\
16.993 \text { Teil- } \\
\text { nehmer }\end{array}$ & $\begin{array}{l}\text { Patientenzufriedenheit: } \\
\text { Standardisierte Mittelwert- } \\
\text { differenz 0,08 mit } \mathrm{Cl}_{95 \%} \\
0,01 \text { bis } 0,15\end{array}$ & Mittel & \multirow{2}{*}{$\begin{array}{l}\text { Die } \\
\text { Patientenzufriedenheit } \\
\text { ist ebenso wie die } \\
\text { Lebensqualität in der } \\
\text { pflegerisch geführten } \\
\text { Primärversorgung } \\
\text { wahrscheinlich etwas } \\
\text { höher. }\end{array}$} \\
\hline & $\begin{array}{l}6 \text { Studien, } \\
16.002 \text { Teil- } \\
\text { nehmer }\end{array}$ & $\begin{array}{l}\text { Lebensqualität: Standardi- } \\
\text { sierte Mittelwertdifferenz } \\
0,16 \text { mit } \mathrm{Cl}_{95 \%} 0,00 \text { bis 0,31 }\end{array}$ & Niedrig & \\
\hline \multirow[t]{3}{*}{$\begin{array}{l}\text { Martinez- } \\
\text { Gonzalez } \\
\text { et al. (2014) }\end{array}$} & & $\begin{array}{l}\text { Patientenzufriedenheit: } \\
\text { Standardisierte Mittelwert- } \\
\text { differenz } 0,18 \text { mit } \mathrm{Cl}_{95 \%} \\
0,13 \text { bis } 0,23\end{array}$ & \multicolumn{2}{|c|}{$\begin{array}{l}\text { Insgesamt fanden sich höhere Werte für die } \\
\text { Zufriedenheit der Patienten mit der pflege- } \\
\text { rischen Versorgung in RCTs mit einmaligem } \\
\text { Kontakt oder in der Notfallversorgung, bei } \\
\text { kurzem Follow-up (weniger als } 6 \text { Monate) } \\
\text { und in kleinen Studien ( } \leq 200 \text { ). }\end{array}$} \\
\hline & & $\begin{array}{l}\text { Krankenhausaufnahme: } \\
\text { Relatives Risiko } 0,76 \text { mit } \\
\mathrm{Cl}_{95 \%} 0,64 \text { bis } 0,91\end{array}$ & \multirow{2}{*}{\multicolumn{2}{|c|}{$\begin{array}{l}\text { Bei pflegerischer Betreuung konnte das } \\
\text { Risiko einer Krankenhausaufnahme sowie die } \\
\text { Mortalität bei RCTs mit laufender oder } \\
\text { nicht-dringender Versorgung, bei längerem } \\
\text { Follow-up (mindestens } 12 \text { Monate) und bei } \\
\text { größeren RCTs }(\mathrm{N}>200 \text { ) reduziert werden. }\end{array}$}} \\
\hline & & $\begin{array}{l}\text { Mortalität: Relatives Risiko } \\
\text { 0,89 mit } \mathrm{Cl}_{95 \%} 0,84 \text { bis 0,96 }\end{array}$ & & \\
\hline
\end{tabular}

Pflege-Report 2019

höher als bei Ärzten. Es gab nur einen geringen oder gar keinen Unterschied zwischen Pflegenden und Ärzten hinsichtlich der Anzahl der ausgestellten Rezepte, dem Besuch von Unfall- und Notfallstationen, der Anzahl von
Tests und Untersuchungen sowie bei Krankenhauseinweisungen.

Die Autoren kommen zu dem Schluss, dass ausgebildete Pflegekräfte (wie z. B. Nurse Practitioners, Practice Nurses und Registered Nur- 
ses) bei einigen anhaltenden und dringenden körperlichen Beschwerden und bei chronischen Erkrankungen wahrscheinlich eine gleiche oder möglicherweise sogar bessere Versorgungsqualität im Vergleich zu Ärztinnen oder Ärzten bieten und wahrscheinlich gleiche oder bessere Gesundheitsergebnisse für die Patientinnen und Patienten erzielen.

In einer anderen systematischen Übersichtsarbeit mit Meta-Analyse (MartinezGonzalez et al. 2014), in die 24 randomisierte kontrollierte Studien mit insgesamt 38.974 Teilnehmerinnen und Teilnehmern sowie zwei ökonomische Analysen eingeschlossen wurden, konnten ähnliche Ergebnisse gezeigt werden (-Tab. 14.1). Die Ergebnisse schienen bei Nurse Practitioners einheitlicher zu sein als bei Registered Nurses oder Licensed Nurses.

\subsection{Studiengang Evidenzbasierte Pflege}

\subsubsection{Entwicklung und Umsetzung}

Kurz nach Erscheinen der Richtlinie des Gemeinsamen Bundesausschusses (G-BA 2012) plante die Medizinische Fakultät der MartinLuther-Universität Halle-Wittenberg (MLU) ein Modellvorhaben nach $₫ 63$ Abs. 3c SGB V und die Qualifizierung von Pflegestudierenden in der Ausübung heilkundlicher Tätigkeiten. So sollte zum einen das Berufsprofil von Pflegenden im Zuge der Akademisierung ausdifferenziert und zum anderen die interdisziplinäre Ausbildung und zukünftige Zusammenarbeit in der gesundheitlichen Versorgung von Patientinnen und Patienten gestärkt werden. Die schriftliche Genehmigung zur Umsetzung des Modellvorhabens nach $\$ 63$ Abs. 3c SGB V erteilte das Bundesministerium für Gesundheit im Mai 2016.

Der im Wintersemester 2016 gestartete primärqualifizierende Studiengang Evidenzbasierte Pflege an der Medizinischen Fakultät der Martin-Luther-Universität Halle-Wittenberg
(MLU) hat zum Ziel, Kenntnisse und Kompetenzen zu vermitteln, um in der Pflege und heilkundlichen Tätigkeit wissenschaftlich fundiert und methodisch reflektiert zu handeln. Die Studierenden sollen die Kompetenz erlangen, die Pflege verantwortungsvoll, bedarfsgerecht und ausgerichtet an der Komplexität des Versorgungsauftrags differenziert zu planen, effektiv mit Patientinnen und Patienten und ihren Angehörigen zu kommunizieren sowie in der interdisziplinären Zusammenarbeit mit anderen relevanten Berufsgruppen des Gesundheits- und Sozialsystems zielorientiert zu interagieren. Darüber hinaus sollen die Studierenden befähigt werden, pflegerelevante Problemstellungen zu identifizieren, sich kritisch-analytisch mit sozial-, gesundheitsund pflegewissenschaftlichen Theorien und aktuellen Forschungserkenntnissen auseinanderzusetzen sowie wissenschaftlich begründete Lösungsansätze unter Berücksichtigung ethischer Prinzipien zu entwickeln.

Die Absolventinnen und Absolventen des Studiengangs werden über die Anforderungen des KrPflG 2003 hinaus in der Lage sein, die verschiedenen Rollenprofile (Expert/in, Informationsvermittler/in, Berater/in, Coach, CaseManager/in und Innovator/in) ihres zukünftigen Berufs qualifiziert auszufüllen. Die Eckdaten des primärqualifizierenden Bachelorstudiengangs "Evidenzbasierte Pflege“ sind in - Tab. 14.2 angeführt.

Im Studiengang werden aus den fünf diagnosebezogenen Bereichen der heilkundlichen Tätigkeiten (G-BA 2012) Kompetenzen zu Diabetes mellitus Typ 2 und chronischen Wunden bzw. Stoma im Umfang von jeweils $250 \mathrm{~h}$ theoretisch und praktisch vermittelt. Die Lehre bestreiten fachlich einschlägig qualifizierte Ärztinnen und Ärzte, die auch die praktische Ausbildung in Zusammenarbeit mit erfahrenen Praxisanleiterinnen, die als Diabetes- und Wundschwestern weitergebildet sind, übernehmen. Die Studierenden lernen Methoden des Assessments, die Planung einzuleitender Interventionen, die Umsetzung des Therapieplans integriert in das Patienten-, Case- und Überleitungsmanagement (einschließlich der psycho- 
- Tabelle 14.2 Eckdaten des primärqualifizierenden Bachelorstudiengangs „Evidenzbasierte Pflege“

\begin{tabular}{|c|c|}
\hline Merkmale & \\
\hline Zugangsvoraussetzung & Hochschulzugangsberechtigung \\
\hline Aufnahmekapazität & 48 Studienplätze \\
\hline Studiendauer & 8 Semester in Vollzeit \\
\hline Leistungspunkte (ECTS) & $180 \mathrm{LP}$ \\
\hline $\begin{array}{l}\text { Theoretischer und praktischer Unterricht } \\
\text { gemäß KrPflAPrV }\end{array}$ & $2.100 \mathrm{~h}$ \\
\hline Praktische Ausbildung gemäß KrPflAPrV & $2.500 \mathrm{~h}$ \\
\hline Abschlüsse & $\begin{array}{l}\text { Bachelor of Science } \\
\text { Erlaubnis zur Führung der Berufsbezeichnung "Gesundheits- } \\
\text { und Krankenpfleger/-in" } \\
\text { Erlaubnis, heilkundliche Tätigkeiten auszuüben }\end{array}$ \\
\hline Besonderheiten & $\begin{array}{l}\text { Starke wissenschaftliche Fundierung } \\
\text { Interprofessionelle Lehre in vielen Modulen } \\
\text { Skills Lab und Simulationszentrum für theoretische Anleitungen } \\
\text { und praktische Übungen } \\
\text { Theoretische Lehre und praktische Anleitung } \\
\text { in den Heilkundemodulen im Umfang von } 500 \mathrm{~h} \\
\text { Eigene schriftliche, mündliche und praktische Prüfungen } \\
\text { in den Bereichen der heilkundlichen Tätigkeiten }\end{array}$ \\
\hline
\end{tabular}

Pflege-Report 2019

sozialen Versorgung) sowie die Evaluation des Therapieplans.

\subsubsection{Qualitätssicherung}

Der Studiengang wird routinemäßig kontinuierlich evaluiert: Neben den Rückmeldungen der Studierenden (jedes Modul in jedem Semester) werden zu Beginn jedes Semesters Modul- und Lehrkonferenzen abgehalten, um einerseits die Inhalte innerhalb der Module und andererseits die Lehre zwischen den Modulen abzustimmen. Zur fachlichen und organisatorischen Planung wurde eine Steuergruppe gegründet, die aus Vertreterinnen und Vertretern des Instituts, des Studiendekanats, des Ausbildungszentrums und des Klinikums besteht und sich in engen Abständen trifft.

Wegen seines Modellcharakters hinsichtlich der heilkundlichen Tätigkeiten wird der
Studiengang von einem externen, unabhängigen Institut intermittierend evaluiert, wobei es geplant ist, die Studierenden auch nach Studienabschluss zu ihrem Verbleib zu befragen. Eine Evaluationskommission und ein externer Fachbeirat begleiten seit Beginn die Umsetzung des Modellstudiengangs.

\subsection{Einmündung in die Gesundheitsversorgung}

Die Universitätsmedizin Halle hat wie jede andere ausbildende Institution im Gesundheitswesen das Bestreben, die Absolventinnen und Absolventen des Studiengangs für eine zukünftige Tätigkeit in der eigenen Einrichtung zu gewinnen. Daher wurde ein Konzeptpapier über die Einmündung in spezifische berufliche Rollen im Klinikum erstellt und es wurden Gehaltsstufen definiert. Die außerklinischen Rol- 
len sind jedoch weit weniger prädefiniert. Hier melden niedergelassene Ärztinnen und Ärzte bereits Interesse an, die Studierenden im Rahmen von Praktika kennenzulernen und zukünftige Beschäftigungsmöglichkeiten zu eröffnen.

Denkbar wäre zukünftig auch eine selbstständige Tätigkeit im Rahmen eines Medizinischen Versorgungszentrums (MVZ) oder einer Berufsausführungsgemeinschaft (BAG). Bisher eröffnet das SGB V allerdings nicht die Möglichkeit für Pflegende, als gleichberechtigte Partner in einem MVZ oder einer BAG tätig zu sein; gegen eine Anstellung unter ärztlicher Leitung hingegen spricht sicher nichts.

Diabetes Typ 2 und chronische Wunden sind häufige Gesundheitsbeeinträchtigungen in Pflegeheimen. Gut vorstellbar ist daher auch eine Tätigkeit der Absolventinnen und Absolventen im Kontext des $\$ 119 \mathrm{~b}$ zur Erfüllung der ambulanten Behandlung in stationären Pflegeeinrichtungen - hier ist eine Position in ambulanten Pflegediensten als Team-Mitglied mit erweiterten Kompetenzen denkbar. Heilkundliche Kompetenz in diesem Bereich, die von Pflegenden ausgeübt wird, könnte eine verbesserte Versorgungssituation der Langzeitpflegebedürftigen in Aussicht stellen. Um einen solchen Effekt zu belegen, wäre es geboten, die klinischen Auswirkungen zu evaluieren. International gibt es vergleichbare berufliche Rollen für Pflegende, etwa die aufsuchend tätige Gerontology Nurse, die ihre erweiterte Kompetenz in der Langzeitpflege zum Einsatz bringt. Ziel ist es, Versorgungsbrüche zu vermeiden, Fehlversorgung von Menschen mit komplexer Multimorbidität $\mathrm{zu}$ begegnen und vor allem auch vermeidbare Krankenhauseinweisungen abzuwenden (Boyd et al. 2014; King et al. 2018).

Andere Konzepte wie die pflegegestützte Niederlassung mit temporärer und/oder telemedizinischer ärztlicher Konsultation wären aussichtsreiche Modelle, um die Versorgung in entlegenen Bereichen sicherzustellen. Selbstverständlich muss ein solches Modell auf Wirksamkeit, Sicherheit und gesundheitsökonomische Auswirkungen evaluiert werden.
In vielen Ländern ist die Implementierung des neuen Skill Mix, also die Mischung aus Gesundheitsberufen sowie die Mischung ihrer Fähigkeiten und Rollen, bereits weit fortgeschritten (Freund et al. 2015; Maier et al. 2018a; Maier et al. 2018b); dies zeigt sich unter anderem daran, dass Pflegende teilweise die erste Anlaufstelle für Patientinnen und Patienten (mit speziellen Erkrankungen) sind (Grant et al. 2017; Maier und Aiken 2016), selbstständig behandeln und überweisen dürfen (Maier und Aiken 2016) oder beispielsweise als Doctor of Nursing Practice noch weiterreichende Kompetenzen erwerben (Walker und Polancich 2015). Deutschland kann hier von den Erfahrungen anderer europäischer Länder wie den Niederlanden, Großbritannien, Irland und Finnland bei der Entwicklung entsprechender Versorgungsmodelle sehr profitieren.

In Deutschland bleiben insbesondere regulatorische Barrieren zu beseitigen: ein „Allgemeines Heilberufegesetz" (Robert-Bosch-Stiftung 2013) könnte a) die erforderliche gesetzliche Grundlage für die unterschiedlich zugeteilten Aufgaben und Tätigkeitsbereiche von Pflegenden und Pflegenden mit heilkundlicher Kompetenz schaffen; b) eine eigenständige Leistungserbringung festschreiben, für die eine entsprechende Vergütung geregelt ist; und c) die Kooperation zwischen Pflegenden und anderen Gesundheitsberufen - auch haftungsrechtlich regeln.

Erweiterte Kompetenzen in der Heilkunde könnten darüber hinaus für die Tätigkeitsbereiche der Rehabilitation und Palliation (RobertBosch-Stiftung 2013) definiert werden und so ermöglichen, dass mehrere Berufsgruppen „entsprechend dem Gedanken von Poolkompetenz" (SVR 2007) situationsabhängig und flexibel die heilkundliche Versorgung sicherstellen können.

Auch wenn die Substitution zu einem verbesserten Einsatz personeller und finanzieller Ressourcen führen kann, fehlen bundesweit bisher weitere Modellvorhaben, in denen qualifikationsentsprechende Regelungen zur Vergütung berücksichtigt werden. 
Erweiterte berufliche Rollenprofile können die Attraktivität des Pflegeberufs steigern, eröffnen Karriereoptionen und könnten in der Summe den Pflegepersonalmangel reduzieren (Robert-Bosch-Stiftung 2018). Bisher sind selbst die Vorteile eines Pflegestudiums, das für die pflegerische Praxis qualifiziert, potenziellen Bewerberinnen und Bewerbern und anderen Berufsgruppen schwer zu vermitteln, da weder eine kritische Anzahl Pflegender mit Bachelorabschluss klinisch tätig ist (Tannen et al. 2017) noch die beruflichen Rollen in den Einrichtungen verbindlich definiert sind. Neue Rollenzuschreibungen für Pflegende mit erweiterten Kompetenzen müssen zukünftig noch unter Berücksichtigung der Versorgungsschnittstellen formuliert werden.

Das neue Pflegeberufegesetz sieht zwar das Studium als regelhafte Option vor - auch für die Vermittlung von erweiterten bzw. heilkundlichen Kompetenzen -, eine Lösung zur Finanzierung der Studiengänge ist jedoch nicht vorgesehen. Darüber hinaus fehlt die Förderung akademischer Praxisanleitung, die ein Praxisumfeld für Studierende zur Sicherung der Ausbildungsqualität vorhalten muss. Somit ist es wahrscheinlich und zugleich bedauerlich, dass die hochschulische Ausbildung von Pflegefachfrauen und -männern in absehbarer Zeit nicht das Nischendasein verlassen wird, das sie bisher zwangsläufig führt.

\section{Literatur}

Boyd M, Armstrong D, Parker J, Pilcher C, Zhou L, McKenzie-Green B et al (2014) Do gerontology nurse specialists make a difference in hospitalization of long-term care residents? Results of a randomized comparison trial. J Am Geriatr Soc 62(10):1962-1967

Bundesministerium für Gesundheit (2012) Bekanntmachung eines Beschlusses des Gemeinsamen Bundesausschusses über eine Richtlinie über die Festlegung ärztlicher Tätigkeiten zur Übertragung auf Berufsangehörige der Alten- und Krankenpflege zur selbständigen Ausübung von Heilkunde im Rahmen von Modellvorhaben nach $\S 63$ Absatz $3 c$ des Fünften Buches Sozialgesetzbuch (SGB V) (Richtlinie nach § 63 Absatz 3c SGB V). BAnz(46):1128
Deutsche Hochschulmedizin e. V. (2014) Universitätsmedizin braucht Finanzierung für Aufbau akademischer Ausbildungen in der Pflege und anderen nicht-ärztlichen Gesundheitsberufen. Stellungnahme des Verbands der Universitätsklinika (VUD), des Verbands der PflegedirektorInnen und Pflegedirektoren der Universitätskliniken und Medizinischen Hochschulen Deutschlands (VPU) und des MFT Medizinischen Fakultätentages

Freund T, Everett C, Griffiths P, Hudon C, Naccarella L, Laurant $M$ (2015) Skill mix, roles and remuneration in the primary care workforce: who are the healthcare professionals in the primary care teams across the world? Int J Nurs Stud 52(3):727-743

Gemeinsamer Bundesauschuss (G-BA) (2012) Richtlinie des Gemeinsamen Bundesauschusses über die Festlegung ärztlicher Tätigkeiten zur Übertragung auf Berufsangehörige der Alten- und Krankenpflege zur selbständigen Ausübung von Heilkunde im Rahmen von Modellvorhaben nach § 63 Abs. 3c SGB V (Richtlinie nach $\S 63$ Abs. 3c SGB V) in der Fassung vom 20. Oktober 2011, veröffentlicht im Bundesanzeiger Nr. 46 (S 1.128) vom 21. März 2012 und Nr. 50 (S 1.228) vom 28. März 2012, in Kraft getreten am 22. März 2012

Grant J, Lines L, Darbyshire P, Parry Y (2017) How do nurse practitioners work in primary health care settings? A scoping review. Int J Nurs Stud 75:51-57

HeilprG (1939) Gesetz über die berufsmäßige Ausübung der Heilkunde ohne Bestallung (Heilpraktikergesetz)

Igl G (2010) Welche rechtlichen Möglichkeiten und Grenzen bestehen in Bezug auf interdisziplinäre Kooperation und Aufgabenverschiebung unter den Gesundheitsberufen? Gutachten erstellt im Auftrag der Robert-Bosch-Stiftung

KfPflG (2003) Krankenpflegegesetz vom 16. Juli 2003 (BGBI I S 1.442), das zuletzt durch Artikel 35 des Gesetzes vom 6. Dezember 2011 (BGBI I S 2.515) geändert worden ist

King All, Boyd ML, Dagley L, Raphael DL (2018) Implementation of a gerontology nurse specialist role in primary health care: Health professional and older adult perspectives. J Clin Nurs 27(3-4):807-818

KrPflAPrV (2003) Ausbildungs- und Prüfungsverordnung für die Berufe in der Krankenpflege

Laurant M, van der Biezen M, Wijers N, Watananirun K, Kontopantelis E, van Vught AJ (2018) Nurses as substitutes for doctors in primary care. Cochrane Database Syst Rev. https://doi.org/10.1002/14651858. CD001271.pub3

Maier CB, Aiken LH (2016) Task shifting from physicians to nurses in primary care in 39 countries: a cross-country comparative study. Eur J Public Health 26(6):927-934 
Maier CB, Barnes H, Aiken LH, Busse R (2016) Descriptive, cross-country analysis of the nurse practitioner workforce in six countries: size, growth, physician substitution potential. BMJ Open 6(9):e11901

Maier CB, Batenburg R, Birch S, Zander B, Elliott R, Busse R (2018a) Health workforce planning: which countries include nurse practitioners and physician assistants and to what effect? Health Policy (New York) 122(10):1085-1092

Maier CB, Budde H, Buchan J (2018b) Nurses in expanded roles to strengthen community-based health promotion and chronic care: policy implications from an international perspective; A commentary. Isr J Health Policy Res 7(1):64

Martinez-Gonzalez NA, Djalali S, Tandjung R, HuberGeismann F, Markun S, Wensing MT et al (2014) Substitution of physicians by nurses in primary care: a systematic review and meta-analysis. BMC Health Serv Res 14:214

Meyer G (2018) Gut abgestimmt - Interdisziplinäre Teams in der Versorgung. „Wir arbeiten konsequent wissensbasiert" Interview mit Gabriele Meyer. G \& G Spezial(3):7

Robert-Bosch-Stiftung (2013) Gesundheitsberufe neu denken, Gesundheitsberufe neu regeln. Grundsätze und Perspektiven - Eine Denkschrift der RobertBosch-Stiftung. Robert-Bosch-Stiftung, Stuttgart
Robert-Bosch-Stiftung (2018) Mit Eliten pflegen. Für eine exzellente, zukunftsfähige Gesundheitsversorgung in Deutschland. Robert-Bosch-Stiftung, Stuttgart

Sachverständigenrat (SVR) (2007) Gutachten des Sachverständigenrates zur Begutachtung des Gesundheitswesens. Kooperation und Verantwortung - Voraussetzungen einer zielorientierten Gesundheitsversorgung. Deutscher Bundestag, Drucksache $16 / 6339$

Sachverständigenrat (SVR) (2014) Gutachten des Sachverständigenrates zur Begutachtung des Gesundheitswesens. Bedarfsgerechte Versorgung - Perspektiven für ländliche Regionen und ausgewählte Leistungsbereiche

Tannen A, Feuchtinger J, Strohbucker B, Kocks A (2017) Survey zur Einbindung von Pflegefachpersonen mit Hochschulabschlüssen an deutschen Universitätskliniken - Stand 2015. Z Evid Fortbild Qual Gesundhwes 120:39-46

Walker DK, Polancich S (2015) Doctor of nursing practice: the role of the advanced practice nurse. Semin Oncol Nurs 31(4):263-272

Wissenschaftsrat (2012) Empfehlungen zu hochschulischen Qualifikationen für das Gesundheitswesen. Drucksache 2411-12. Wissenschaftsrat, Berlin

Open Access Dieses Kapitel wird unter der Creative Commons Namensnennung 4.0 International Lizenz (http:// creativecommons.org/licenses/by/4.0/deed.de) veröffentlicht, welche die Nutzung, Vervielfältigung, Bearbeitung, Verbreitung und Wiedergabe in jeglichem Medium und Format erlaubt, sofern Sie den/die ursprünglichen Autor(en) und die Quelle ordnungsgemäß nennen, einen Link zur Creative Commons Lizenz beifügen und angeben, ob Änderungen vorgenommen wurden.

Die in diesem Kapitel enthaltenen Bilder und sonstiges Drittmaterial unterliegen ebenfalls der genannten Creative Commons Lizenz, sofern sich aus der Abbildungslegende nichts anderes ergibt. Sofern das betreffende Material nicht unter der genannten Creative Commons Lizenz steht und die betreffende Handlung nicht nach gesetzlichen Vorschriften erlaubt ist, ist für die oben aufgeführten Weiterverwendungen des Materials die Einwilligung des jeweiligen Rechteinhabers einzuholen. 